TOKYO J. MATH.

VOL. 36, No. 2, 2013

\title{
Pseudo-Anosov Maps and Pairs of Filling Simple Closed Geodesics on Riemann Surfaces, II
}

\author{
Chaohui ZHANG
}

Morehouse College

(Communicated by M. Tanaka)

\begin{abstract}
Let $S$ be a Riemann surface containing at least two punctures $z$ and $z_{0}$. Let $\mathscr{F}(S)$ be the set of pseudo-Anosov maps of $S$ that are isotopic to the identity on $S \cup\{z\}$. We show that for any $f \in \mathscr{F}(S)$ and any twice punctured disk $\Delta$ enclosing $z$ and $z_{0}$, the pair $(\partial \Delta, f(\partial \Delta))$ fills $S$, where $\partial \Delta$ denotes the boundary of $\Delta$. Fix such a $\Delta$, and denote by $\mathscr{T}(\Delta)$ the set of twice punctured disks $\Delta^{\prime}$ on $S$ enclosing $z$ and $z_{0}$ with the property that $\left(\partial \Delta, \partial \Delta^{\prime}\right)$ fills $S$. Let $\Delta_{0} \in \mathscr{T}(\Delta)$. We describe all possible pseudo-Anosov maps $f$ in $\mathscr{F}(S)$ sending $\Delta$ to $\Delta_{0}$, and classify elements of $\mathscr{F}(S)$ in terms of $\mathscr{T}(\Delta)$. We also show that there are infinitely many elements $f_{k} \in \mathscr{F}(S)$ with $f_{k}(\Delta)=\Delta_{0}$ such that their dilatations $\lambda\left(f_{k}\right) \rightarrow+\infty$ as $k \rightarrow+\infty$.
\end{abstract}

\section{Introduction and statement of results}

Let $S$ be an analytically finite Riemann surface of type $(p, n)$ with $3 p+n>3$, where $p$ is the genus and $n$ is the number of punctures of $S$. For any pseudo-Anosov map $f: S \rightarrow S$, and any simple closed geodesic $a \subset S$ (with respect to a hyperbolic metric on $S$, of course), the set $\mathscr{S}=\left\{a, f(a), f^{2}(a), \ldots\right\}$ fills $S$ in the sense that each closed geodesic on $S$ intersects one of the elements in $\mathscr{S}$ (see $[6,7]$ ), where and below $f^{i}(a)$ denotes the geodesic representative in the homotopy class of the image curve of $a$ under $f^{i}$. In [11] Masur-Minsky showed that $\left(a, f^{k}(a)\right)$ fills $S$ for all sufficiently large integers $k$.

Consider the case where $3 p+n>4$ and $n \geq 1$. Let $z$ denote a puncture of $S$. Write $\tilde{S}=S \cup\{z\}$. Let $c \subset S$ be a simple closed geodesic. Then $c$ can also be viewed as a curve $\tilde{c}$ on $\tilde{S}$. Note that $\tilde{c}$ could be trivial, that is, $\tilde{c}$ could be homotopic to a puncture of $\tilde{S}$. If this occurs, then $c$ bounds a (topological) twice punctured disk on $S$ enclosing $z$ and another puncture of $S$. See Fig. 1 (a) and (b) for examples of twice punctured disks. It is clear that no such geodesic exists when $n=1$. If $n \geq 2$, there are infinitely many non-trivial geodesics on $S$ that are trivial on $\tilde{S}$. When $\tilde{c}$ is non-trivial, there is a unique geodesic representative in the homotopy class of $\tilde{c}$. For simplicity, we call this geodesic representative $\tilde{c}$ also.

Received February 2, 2012; revised February 1, 2013

Mathematics Subject Classification: 53C35, 53C40

Key words and phrases: Riemann surfaces, Dehn twists, pseudo-Anosov maps, twice punctured disks, filling and simple closed geodesics 
Let $\mathscr{F}_{0}(S)$ be the set consisting of mapping classes on $S$ that fix $z$ and are isotopic to the identity on $\tilde{S}$ as $z$ is filled in. Let $\mathscr{F}(S)$ be the subset of $\mathscr{F}_{0}(S)$ consisting of pseudo-Anosov elements. It was shown in [21] that for any $f \in \mathscr{F}(S)$, and any simple closed geodesic $a$ with $\tilde{a}$ being non-trivial on $\tilde{S},\left(a, f^{k}(a)\right)$ fills $S$ for all $k \geq 3$. In this article, we consider the set of geodesics that are boundaries of twice punctured disks, which is identified with the set of geodesics $b$ with $\tilde{b}$ being trivial on $\tilde{S}$.

Throughout the article we assume that $S$ contains at least two punctures $z$ and $z_{0}$. We first prove the following result.

THEOREM 1.1. With the above assumptions, let $\Delta$ be a twice punctured disk on $S$ that encloses $z$ and $z_{0}$. Then for any $f \in \mathscr{F}(S),\left(\partial \Delta, \partial f^{k}(\Delta)\right)$ fills $S$ for all $k \geq 1$.

The converse is not true. Let $t_{c}$ denote the positive Dehn twist along a simple closed geodesic $c$. We know that there is a geodesic $c$ and thus a Dehn twist $t_{c}$ such that both pairs $(\partial \Delta, c)$ and $\left(\partial \Delta, t_{c}(\partial \Delta)\right)$ fill $S$ (See [16] for constructions). In Section 7, we will acquire some spin maps $t_{c} \circ t_{c_{0}}^{-1}$ on $S$ such that $\left(\partial \Delta, t_{c} \circ t_{c_{0}}^{-1}(\partial \Delta)\right)$ fill $S$. Theorem 1.1 can be extended to the following corollary.

COROLlARY 1.1. Let $\alpha \subset S$ be a simple closed geodesic which bounds a planar region $D_{\alpha}$ enclosing $z$ and at least one more puncture of $\tilde{S}$. Then for every $f \in \mathscr{F}(S)$, $(\alpha, f(\alpha))$ fills $S$.

Let $\Delta$ be a fixed twice punctured disk that encloses $z$ and $z_{0}$. Note that $z_{0}$ is also a puncture on $\tilde{S}$. Let $\mathscr{T}(\Delta)$ be the set of twice punctured disks $\Delta_{0}$ enclosing $z$ and $z_{0}$ with geodesic boundaries such that $\left(\partial \Delta, \partial \Delta_{0}\right)$ fills $S$. There are infinitely many elements in $\mathscr{T}(\Delta)$ (see [18]).

THEOREM 1.2. Let $S$ be as above. Then for any $\Delta_{0} \in \mathscr{T}(\Delta)$, there is $F \in \mathscr{F}_{0}(S)$ such that $F(\Delta)=\Delta_{0}$. Furthermore, by suitably choosing $\varepsilon=1$ or -1 , the maps $F \circ t_{\partial \Delta}^{\varepsilon k}$ are pseudo-Anosov for any $k>0$ and send $\Delta$ to $\Delta_{0}$.

It should be noted that in Theorem 1.2 we do not assume $F$ is pseudo-Anosov, and only assume that the image $F(\partial \Delta)$ along with $\partial \Delta$ fills $S$ (if $F$ is pseudo-Anosov, the theorem was proved in [22]).

For a general pseudo-Anosov map $f$ and a Dehn twist $t_{c}$ for a simple geodesic $c$, LongMorton [12] proved that $f \circ t_{c}^{k}$ are pseudo-Anosov except for at most $N(<\infty)$ consecutive integer values of $k$. Fathi [6] showed that $N \leq 7$, and later Boyer et al. [5] showed that $N \leq 6$. During the course of the proof of Theorem 1.2, we describe the condition which guarantees that $F \circ t_{\partial \Delta}^{k}$ are pseudo-Anosov for all $k>0$ or $k<0$. Of course, our method is different from those used in $[5,6,12]$.

Let $\mathbf{D}$ denote the unit disk equipped with the hyperbolic metric $2|d z| /\left(1-|z|^{2}\right)$, and let $\varrho: \mathbf{D} \rightarrow \tilde{S}$ denote the universal covering map with a covering group $G$ which is isomorphic to the fundamental group $\pi_{1}(\tilde{S}, z)$. It is well known [10] that for each $\Delta^{\prime} \in \mathscr{T}(\Delta)$, there 
are parabolic elements $T, T^{\prime} \in G$ that correspond to $t_{\partial \Delta}$ and $t_{\partial \Delta^{\prime}}$, respectively, under the Bers isomorphism $\varphi$ (see Section 2 for expositions). Note that $\Delta$ and $\Delta^{\prime}$ enclose the same punctures $z$ and $z_{0}$. Hence $T$ is conjugate to $T^{\prime}$ in $G$, which means that there is an element $h \in G$ that sends the fixed point of $T$ to the fixed point of $T^{\prime}$. Let $h^{*}$ be the corresponding element in $\mathscr{F}_{0}(S)$. By combining Theorem 1.2 we can obtain the following corollary.

COROLlaRY 1.2. Let $\Delta, \Delta^{\prime}$ be any twice punctured disks enclosing $z$. Then there is $f \in \mathscr{F}(S)$ sending $\Delta$ to $\Delta^{\prime}$ if and only if $\Delta^{\prime} \in \mathscr{T}(\Delta)$.

It is well known $[2,4]$ that $\mathscr{F}_{0}(S)$ is isomorphic to $\pi_{1}(\tilde{S}, z)$ and that there is a bijection between $\mathscr{F}(S)$ and the set of essential hyperbolic elements of $G$, where an element $g \in G$ is called an essential hyperbolic if it is hyperbolic and its axis axis $(g)$ projects to a filling closed geodesic $\tilde{\gamma}$ in the sense that $\tilde{\gamma}$ intersects every simple closed geodesic on $\tilde{S}$. Moreover, the set of conjugacy classes of elements of $\mathscr{F}(S)$ in $\mathscr{F}_{0}(S)$ is one-to-one correspondent with the set of oriented primitive filling closed geodesics on $\tilde{S}$.

Two elements $f, f^{\prime} \in \mathscr{F}(S)$ are said to be $\Delta$-equivalent (denoted by $f \sim f^{\prime}$ ) if $f=$ $f^{\prime} \circ t_{\partial \Delta}^{k}$ for an integer $k$. It is obvious that " $\sim$ " is an equivalent relation. Our next result gives a new characterization of equivalence classes of elements of $\mathscr{F}(S)$ by means of twice punctured disks on $S$.

THEOREM 1.3. There is a bijection between $\mathscr{F}(S) / \sim$ and $\mathscr{T}(\Delta)$.

In [9], Harvey introduced a complex $\mathcal{C}(S)$ of curves on $S$. A $k$-th dimensional simplex of $\mathcal{C}(S)$ is a collection of $k+1$ disjoint simple closed geodesics on $S$. In particular, the vertices $\mathcal{C}_{0}$ of $\mathcal{C}(S)$ are collections of simple closed geodesics on $S$. We define the length of each edge in $\mathcal{C}_{1}$ is one, and define the distance $d_{\mathcal{C}}(a, b)$ between two vertices $a, b \in \mathcal{C}_{0}$ to be the least number of edges in $\mathcal{C}_{1}$ joining $a$ and $b$. By the definition, we know that $d_{\mathcal{C}}(a, b) \geq 3$ if and only if $(a, b)$ fills $S$. Also, $d_{\mathcal{C}}(a, b)=1$ if and only if $a$ and $b$ are disjoint. Thus, for any $\Delta_{0}, \Delta_{1} \in \mathscr{T}(\Delta), d_{\mathcal{C}}\left(\partial \Delta_{0}, \partial \Delta_{1}\right)>1$ and Theorem 1.1 says that $d_{\mathcal{C}}(\partial \Delta, f(\partial \Delta)) \geq 3$ for any $f \in \mathscr{F}(S)$.

In [23], we considered vertices $a_{1}, a_{2} \in \mathcal{C}_{0}$ that are non-trivial and are homotopic to each other on $\tilde{S}$, and proved that if $d_{\mathcal{C}}\left(a_{1}, a_{2}\right) \geq 3$, there is a sequence $f_{k} \in \mathscr{F}(S)$ such that $f_{k}\left(a_{1}\right)=a_{2}$ while their dilatations $\lambda\left(f_{k}\right)$ tend to infinity. Here we treat the case in which $a_{1}, a_{2} \in \mathscr{T}(\Delta)$ :

THEOREM 1.4. Let $\Delta$ be a twice punctured disk on $S$ enclosing $z$ and another puncture $z_{0}$ of $S$.

(1) For any $\Delta_{0} \in \mathscr{T}(\Delta)$, any large integer $M$, there are $f \in \mathscr{F}(S)$ such that $f(\Delta)=$ $\Delta_{0}$ and $\lambda(f)>M$.

(2) Let $\Delta_{k} \in \mathscr{T}(\Delta)$ be such that $d_{\mathcal{C}}\left(\partial \Delta, \partial \Delta_{k}\right) \rightarrow+\infty$ as $k \rightarrow+\infty$. Then for any elements $f_{k}: \Delta \rightarrow \Delta_{k}$ of $\mathscr{F}(S)$, the sequence $\left\{\lambda\left(f_{k}\right)\right\}$ is unbounded.

This article is organized as follows. In Section 2, we collect background materials on zpointed mapping class group $\operatorname{Mod}_{S}^{z}$. Some special elements in $\operatorname{Mod}_{S}^{z}$ and their combinations 
are investigated. In Section 3, we prove Theorem 1.1. In Section 4, we prove Theorem 1.2. In Section 5, we classify elements of $\mathscr{F}(S)$ in terms of $\mathscr{T}(\Delta)$ and prove Theorem 1.3. In Section 6, we study the relationship between the path distance $d_{\mathcal{C}}\left(\partial \Delta, \partial \Delta_{k}\right)$ for any $\Delta_{k} \in \mathscr{T}(\Delta)$ and the dilatation of any associated pseudo-Anosov maps obtained from Theorem 1.2, and prove Theorem 1.4. In Section 7, we illustrate that for a filling pair $\left(\partial \Delta, \partial \Delta_{0}\right)$ with $\partial \Delta_{0}=\partial f(\Delta)$, the maps $f$ may not be pseudo-Anosov. We give some examples showing that $f$ could stem from parabolic or simple hyperbolic elements of $G$.

\section{Background and some preliminary results}

Let $G$ be the covering group of a holomorphic universal covering map $\varrho: \mathbf{D} \rightarrow \tilde{S}$. Then $G$ is a torsion free finitely generated Fuchsian group of the first kind. Elements of $G$ are either parabolic or hyperbolic and are isometric motions on $\mathbf{D}$ with respect to the hyperbolic metric on D. Let $Q(G)$ be the group of quasiconformal automorphisms $w$ of $\mathbf{D}$ such that $w G w^{-1}=G$. Two maps $w, w_{0} \in Q(G)$ are said to be equivalent (denoted by $w \sim w_{0}$ ) if $\left.w\right|_{\mathbf{S}^{1}}=\left.w_{0}\right|_{\mathbf{S}^{1}}$. Denote by $[w]$ the equivalence class of $w$. Thus the restriction $\left.[w]\right|_{\mathbf{S}^{1}}$ is well defined and is a quasisymmetric map on the unit circle $\mathbf{S}^{1}$. By the Bers isomorphism theorem [2], the quotient group $Q(G) / \sim$ is isomorphic to the $z$-pointed mapping class group $\operatorname{Mod}_{S}^{z}$ that consists of mapping classes $f$ with $f(z)=z$.

According to the Nielsen-Thurston classification for surface homeomorphisms [14], every non-periodic element of $\operatorname{Mod}_{S}^{z}$ is either reducible or pseudo-Anosov, where by a reducible mapping class $f$ we mean that there is a representative of the mapping class (also denoted by $f$ ) and a curve simplex

$$
\Gamma=\left\{u_{1}, \ldots, u_{s}\right\}, \quad s \geq 1,
$$

such that $f\left(\left\{u_{1}, \ldots, u_{s}\right\}\right)=\left\{u_{1}, \ldots, u_{s}\right\}$; and by a pseudo-Anosov mapping class $f$ we mean that there is a representative (denoted by $f$ also), a pair $\left(\mathcal{F}_{+}, \mathcal{F}_{-}\right)$of transverse measured foliations and a real number $\lambda>1$ such that $f\left(\mathcal{F}_{+}\right)=\lambda \mathcal{F}_{+}$and $f\left(\mathcal{F}_{-}\right)=(1 / \lambda) \mathcal{F}_{-}$. The number $\lambda=\lambda(f)$ is called the dilatation of $f$.

Let $w \in Q(G)$ be such that $[w]$ corresponds to $f$ under the Bers isomorphism. As all elements of $\operatorname{Mod}_{S}^{z}$ fix $z$, it is clear that there defines a group homomorphism of $\operatorname{Mod}_{S}^{z}$ onto the ordinary mapping class group $\operatorname{Mod}(\tilde{S})$ by sending every element $f \in \operatorname{Mod}_{S}^{z}$ to an element of $\operatorname{Mod}(\tilde{S})$ induced by a homeomorphism $\tilde{f}$ of $\tilde{S}$, where $\tilde{f}$ can also be obtained from the projection of the map $w$ via the universal covering map $\varrho$.

In what follows, for each $w \in Q(G)$, we denote by $[w]^{*} \in \operatorname{Mod}_{S}^{z}$ the corresponding element under the Bers isomorphism. In particular, as $G$ is considered a normal subgroup of $Q(G) / \sim$, we use the symbol $h^{*}$, where $h \in G$, to denote the mapping class in $\operatorname{Mod}_{S}^{z}$ as well as a homeomorphism representing $h^{*}$.

We proceed to investigate mapping classes $h^{*}$ for elements $h \in G$. Details can be found in Kra [10]. In the case where $h$ is parabolic, $h^{*}$ is the Dehn twist $t_{\partial \Delta}$ or its inverse $t_{\partial \Delta}^{-1}$ along 
$\partial \Delta$ for a twice punctured disk $\Delta$ enclosing $z$, by which we mean a planar region on $S$ that contains the puncture $z$ and another puncture of $\tilde{S}$. Fig. 1 (a) exhibits an "obvious" twice punctured disk on a surface of type $(2,4)$, which encloses $z$ and $z_{0}$, while Fig. 1 (b) is a highly complicated twice punctured disk on the same surface; it also encloses $z$ and $z_{0}$.

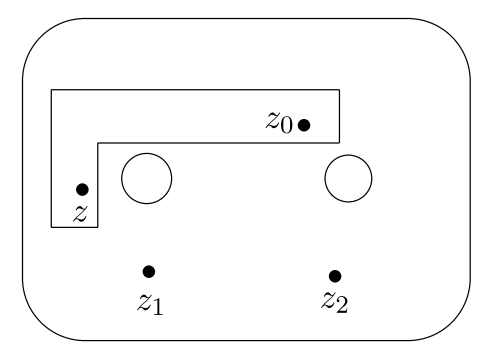

(a)

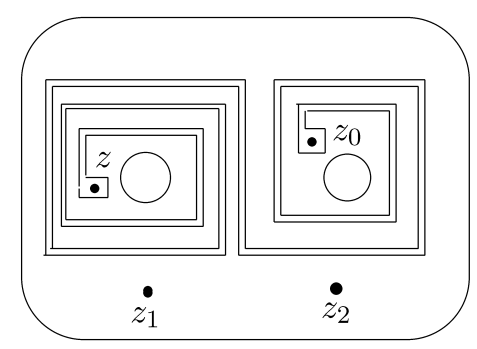

(b)

FIG. 1

Conversely, for any Dehn twist $t_{\partial \Delta}$ along the boundary $\partial \Delta$ of a twice punctured disk $\Delta$ enclosing $z$, there exists a parabolic element $h \in G$ such that $h^{*}=t_{\partial \Delta}$.

If $h$ is simple hyperbolic; that is, its axis axis $(h) \subset \mathbf{D}$ projects to a simple closed geodesic $\varrho(\operatorname{axis}(h)) \subset \tilde{S}$, then there is a pair of simple closed geodesics $\left\{c_{0}, c\right\} \subset S$ that bounds a $z-$ punctured cylinder, such that $h^{*}=t_{c_{0}} \circ t_{c}^{-1}$, and that $\varrho(\operatorname{axis}(h))=\tilde{c}=\tilde{c}_{0}$, where we recall that $\tilde{c}$ is the geodesic representative in the homotopy class of $c$ if $c$ is regarded as a curve on $\tilde{S}$. Conversely, for any $z$-punctured cylinder $\mathscr{P}$ on $S$, there is a simple hyperbolic element $h \in G$ such that $h^{*}=t_{c_{0}} \circ t_{c}^{-1}$ for $\left\{c_{0}, c\right\}=\partial \mathscr{P}$ and $\operatorname{axis}(h)$ projects to $\varrho(\operatorname{axis}(h))=\tilde{c}=\tilde{c}_{0}$.

If $h$ is essential hyperbolic; that is, axis $(h)$ projects to a filling closed geodesic $\varrho(\operatorname{axis}(h))$ on $\tilde{S}$, then $h^{*}$ is pseudo-Anosov and hence $h^{*} \in \mathscr{F}(S)$. By Theorem 2 of [10], all elements of $\mathscr{F}(S)$ can be obtained in this way.

Finally, if $h \in G$ is non-simple and non-essential, i.e., $\varrho(\operatorname{axis}(h))$ is a non-filling selfintersecting closed geodesic on $\tilde{S}$, then $h^{*} \in \mathscr{F}_{0}(S)$ is reducible by a maximal reduced curve simplex (call it $\Gamma$ also). Let $P$ be the component of $S \backslash \Gamma$ that contains the puncture $z$. Then by Theorem 2 of [10], we know that $\left.h^{*}\right|_{S \backslash P}$ is the identity and $\left.h^{*}\right|_{P}$ is pseudo-Anosov. In what follows $P$ is called the pseudo-Anosov component for $h^{*}$.

We also need to explore some special elements in $Q(G) / \sim$ that are different from elements of $G$. Let $u \subset S$ be a simple closed geodesic such that $\tilde{u} \subset \tilde{S}$ is also non-trivial. Let $\left\{\varrho^{-1}(\tilde{u})\right\}$ be the collection of all geodesics $\hat{u}$ in $\mathbf{D}$ such that $\varrho(\hat{u})=\tilde{u}$. Since $\tilde{u}$ is simple, all geodesics in $\left\{\varrho^{-1}(\tilde{u})\right\}$ are mutually disjoint.

Fix a geodesic $\hat{u} \in\left\{\varrho^{-1}(\tilde{u})\right\}$ and fix a component $U$ of $\mathbf{D} \backslash \hat{u}$, there is a lift $\tau_{\hat{u}}$ of the Dehn twist $t_{\tilde{u}}$ with respect to $U$. See $[17,19]$ for more information on the lift $\tau_{\hat{u}}$. It is known that 
$\tau_{\hat{u}} \in Q(G)$ determines a maximal convex region $\Omega_{\hat{u}}$ in $\mathbf{D} \backslash U$ with geodesic boundaries, and that the restriction $\left.\tau_{\hat{u}}\right|_{\Omega_{\hat{u}}}$ is the identity. By Lemma 3.2 of [17], we know that $\hat{u}$ (and hence $U$ ) can be properly chosen so that $\left[\tau_{\hat{u}}\right]^{*}=t_{u}$. Therefore, the pair $(\hat{u}, U)$ completely determines the geodesic $u$.

In fact, $\Omega_{\hat{u}}$ is a component of $\mathbf{D} \backslash\left\{\varrho^{-1}(\tilde{u})\right\}$ that takes $\hat{u}$ as a component of the boundary $\partial \Omega_{\hat{u}}$. The complement of the closure of $\Omega_{\hat{u}}$ are the disjoint union of half-spaces in $\mathbf{D}$, where by a half-space we mean one of the components of a geodesic in $\left\{\varrho^{-1}(\tilde{u})\right\}$ which is disjoint from $\Omega_{\hat{u}}$. By our convention, a half-space $D$ includes the open $\operatorname{arc} D \cap \mathbf{S}^{1}$. Note that the endpoints of this arc are the fixed points of a simple hyperbolic element of $G$. Let $\mathscr{U}_{\hat{u}}$ denote the collection of all half-spaces in $\mathbf{D}$ defined by the geodesics in $\left\{\varrho^{-1}(\tilde{u})\right\}$. We see that $\mathscr{U}_{\hat{u}}$ forms a partially ordered set whose order is defined by inclusion. Each component in the complement of the closure of $\Omega_{\hat{u}}$ is called a maximal element of $\mathscr{U}_{\hat{u}}$. Observe that $\left\{\varrho^{-1}(\tilde{u})\right\}$ contains infinitely many mutually disjoint geodesics and $\Omega_{\hat{u}}$ contains no geodesics in $\left\{\varrho^{-1}(\tilde{u})\right\}$. Every geodesic in $\left\{\varrho^{-1}(\tilde{u})\right\}$, if not the boundary of any maximal element, is included in a maximal element of $\mathscr{U}_{\hat{u}}$. As such, each maximal element contains infinitely many elements of $\mathscr{U}_{\hat{u}}$ of higher orders. Notice that the map $\tau_{\hat{u}}$ leaves invariant each maximal element of $\mathscr{U}_{\hat{u}}$ and sends each element of $\mathscr{U}_{\hat{u}}$ to an element of $\mathscr{U}_{\hat{u}}$ with the same order. In what follows, the triple $\left(\tau_{\hat{u}}, \Omega_{\hat{u}}, \mathscr{U}_{\hat{u}}\right)$ is called a configuration corresponding to the geodesic $u$.

LEMmA 2.1. Let $h \in G$. Then $h$ sends every maximal element of $\mathscr{U}_{\hat{u}}$ to a different maximal element if and only if the fixed point $(s)$ of h lies in $\Omega_{\hat{u}} \cap \mathbf{S}^{1}$.

PROOF. We only prove the case that $h$ is parabolic (the hyperbolic case can be handled similarly). Let $x$ be the fixed point of $h$. If $x \in \Omega_{\hat{u}} \cap \mathbf{S}^{1}$, that is, $x$ lies outside of all maximal elements of $\mathscr{U}_{\hat{u}}$, then by construction, $\tau_{\hat{u}}(x)=x$. Since $\tau_{\hat{u}} \in Q(G), \tau_{\hat{u}} h \tau_{\hat{u}}^{-1}$ is also a primitive parabolic element of $G$ with fixed point $x$. It follows that $\tau_{\hat{u}} h \tau_{\hat{u}}^{-1}=h$, i.e., $\tau_{\hat{u}} h=h \tau_{\hat{u}}$. Hence for each maximal element $U \in \mathscr{U}_{\hat{u}}, h(U)$ is also a maximal element. Conversely, if $x \in U$ for a maximal element $U$ of $\mathscr{U}_{\hat{u}}$, then $x$ lies outside of $\mathbf{D} \backslash U$. By examining the action of $h$ on $\mathbf{D}, h(\mathbf{D} \backslash U)$ is disjoint from $\mathbf{D} \backslash U$. Hence $h(\mathbf{D} \backslash U) \subset U$ and thus $U$ intersects $h(U)$. It follows from Lemma 4.3 of [19] that $h(U)$ is not a maximal element of $\mathscr{U}_{\hat{u}}$.

LEMMA 2.2. Let $\left(\tau_{\hat{u}}, \Omega_{\hat{u}}, \mathscr{U}_{\hat{u}}\right)$ be the configuration corresponding to $u$. Let $x$ be the parabolic fixed point of $G$ that corresponds to a simple closed geodesic $a=\partial \Delta$. Then the geodesic $u$ intersects $a$ if and only if $x$ is covered by a maximal element of $\mathscr{U}_{\hat{u}}$.

PROOF. If $x$ lies outside of any maximal element of $\mathscr{U}_{\hat{u}}$, i.e., $x \in \Omega_{\hat{u}} \cap \mathbf{S}^{1}$, then $\tau_{\hat{u}}(x)=x$. Let $T \in G$ be the primitive parabolic element with the fixed point $x$. By the same argument of Lemma 2.1, $\tau_{\hat{u}} T=T \tau_{\hat{u}}$. Via the Bers isomorphism, we obtain $t_{u} \circ t_{a}=t_{a} \circ t_{u}$, which implies that $u$ and $a$ are disjoint. Conversely, if $u$ is disjoint from $a$, then $\tau_{\hat{u}}$ fixes $x$. So $x \in \Omega_{\hat{u}} \cap \mathbf{S}^{1}$.

From Lemma 2.2, we know that $u$ is disjoint from $\partial \Delta$ if $x$ stays outside of all maximal 
elements of $\mathscr{U}_{\hat{u}}$. Similar situation occurs when $h$ is non-essential hyperbolic with axis axis $(h)$. In this case, there exists a simple closed geodesic $v \subset S$, with $\tilde{v}$ being non-trivial, such that $v$ is disjoint from the pseudo-Anosov component $P$ of $h^{*}$. It follows from Lemma 2.1 that both fixed points of $h$ lie in $\Omega_{\hat{v}} \cap \mathbf{S}^{1}$. As $\Omega_{\hat{v}}$ is convex with geodesic boundary, it is clear that the axis axis $(h)$ of $h$ lies outside of any maximal element of $\mathscr{U}_{\hat{v}}$.

Let $w \in Q(G)$ be such that $[w]^{*} \in \operatorname{Mod}_{S}^{z}$ is a reducible mapping class by a reduced curve simplex $\Gamma$ as defined in (2.1). Note that if $\tilde{u}_{j}$ is a non-trivial geodesic for some $j \in$ $\{1,2, \ldots, s\}$, that is, $u_{j}$, if viewed as a curve on $\tilde{S}$, is homotopic to neither a point nor a puncture of $\tilde{S}$, then, as discussed earlier, there defines a configuration $\left(\tau_{\hat{u}_{j}}, \Omega_{\hat{u}_{j}}, \mathscr{U}_{\hat{u}_{j}}\right)$ that corresponds to $u_{j}$ (in the sense that we can choose the lift $\hat{u}_{j}$ of $\tilde{u}_{j}$ and the component $U$ of $\mathbf{D} \backslash \hat{u}_{j}$ on which the lift $\tau_{\hat{u}_{j}}$ of $t_{\tilde{u}_{j}}$ is constructed). See the discussion above Lemma 2.1.

Note also that any two twice punctured disks, if both enclose $z$, must intersect. Since all elements of $\Gamma$ are disjoint, there is at most one geodesic $u$ in $\Gamma$ such that $\tilde{u}$ is trivial.

LEMMA 2.3. With the above conditions:

(1) If there is a $u_{i} \in \Gamma$ with $\tilde{u}_{i}$ being non-trivial such that $[w]^{*}\left(u_{i}\right)=u_{i}$, Then there is $w_{0} \in Q(G)$ with $w_{0} \sim w$ such that $w_{0}$ sends every maximal element of $\mathscr{U}_{\hat{u}_{i}}$ to a maximal element of $\mathscr{U}_{\hat{u}_{i}}$.

(2) If there are $u_{i}, u_{j} \in \Gamma, i \neq j$, such that $[w]^{*}\left(u_{i}\right)=u_{j}$, then $\tilde{u}_{i}$ and $\tilde{u}_{j}$ are nontrivial and there is $w_{0} \in Q(G)$ with $w_{0} \sim w$ such that $w_{0}$ sends every maximal element of $\mathscr{U}_{\hat{u}_{i}}$ to a maximal element of $\mathscr{U}_{\hat{u}_{j}}$.

Proof. (1) is proved in [19]. For (2), we notice that if $\Gamma$ contains a geodesic $u$ so that $\tilde{u}$ is trivial on $\tilde{S}$, then such a curve $u$ is unique. This tells us that $[w]^{*}(u)=u$. In other words, if $[w]^{*}\left(u_{i}\right)=u_{j}$ for some $u_{i}, u_{j} \in \Gamma$ with $u_{i} \neq u_{j}$, then both $\tilde{u}_{i}$ and $\tilde{u}_{j}$ are non-trivial. Thus the configurations $\left(\tau_{\hat{u}_{i}}, \Omega_{\hat{u}_{i}}, \mathscr{U}_{\hat{u}_{i}}\right)$ and $\left(\tau_{\hat{u}_{j}}, \Omega_{\hat{u}_{j}}, \mathscr{U}_{\hat{u}_{j}}\right)$, which correspond to $u_{i}$ and $u_{j}$, respectively, are defined. The rest of the proof is similar to (1) which was given in [19].

More generally, we have

LEMMA 2.4. Assume that $[w]^{*} \in \operatorname{Mod}_{S}^{z}$ is a reducible mapping class with the reduced curve simplex (2.1). Also assume that each $\tilde{u}_{i}, u_{i} \in \Gamma$, is non-trivial. Then for every maximal element $U_{1} \in \mathscr{U}_{\hat{u}_{1}}, w^{k}\left(U_{1}\right) \cup U_{1} \neq \mathbf{D}$ for all integers $k$.

Proof. Suppose that for an integer $k_{0}$ and a maximal element $U_{1} \in \mathscr{U}_{\hat{u}_{1}}$, we have $w^{k_{0}}\left(U_{1}\right) \cup U_{1}=\mathbf{D}$. If $s=1$, then $[w]^{*}\left(u_{1}\right)=u_{1}$. By Lemma $2.3(1), w$ sends every maximal element $U_{1} \in \mathscr{U}_{\hat{u}_{1}}$ to a maximal element. Since all maximal elements of $\mathscr{U}_{\hat{u}_{1}}$ are disjoint and $\Omega_{\hat{u}_{1}}$ is not empty, we see that $w^{k}\left(U_{1}\right) \cup U_{1} \neq \mathbf{D}$. Thus we assume that $s \geq 2$ and that $\left([w]^{*}\right)^{k_{0}}\left(u_{1}\right)=u_{2}$, where $u_{1}, u_{2} \in \Gamma$. Then $t_{u_{2}}=\left([w]^{*}\right)^{k_{0}} \circ t_{u_{1}} \circ\left([w]^{*}\right)^{-k_{0}}$, which says that $w^{k_{0}} \tau_{\hat{u}_{1}} w^{-k_{0}}=\tau_{\hat{u}_{2}}$. It follows that $w^{k_{0}}\left(\mathscr{U}_{\hat{u}_{1}}\right)$ is the collection of half-spaces defined by $\tau_{\hat{u}_{2}}$ and that $w^{k_{0}}\left(U_{1}\right) \in \mathscr{U}_{\hat{u}_{2}}$ is a maximal element. Since $w^{k_{0}}\left(U_{1}\right) \cup U_{1}=\mathbf{D}$, by Lemma 
4 of [15], we have $\left[\tau_{\hat{u}_{2}}\right]\left[\tau_{\hat{u}_{1}}\right] \neq\left[\tau_{\hat{u}_{1}}\right]\left[\tau_{\hat{u}_{2}}\right]$. Thus $t_{u_{1}} \circ t_{u_{2}} \neq t_{u_{2}} \circ t_{u_{1}}$. Hence $u_{1}$ intersects $u_{2}$, contradicting that $u_{1}, u_{2} \in \Gamma$.

\section{Proof of Theorem 1.1}

For simplicity, write $a=\partial \Delta, b=f(\partial \Delta)$ and $f=g^{*}$ for some essential hyperbolic element $g \in G$. Suppose that $(a, b)$ does not fill $S$. There exists a simple closed geodesic $u$ such that

$$
t_{a}^{r} \circ t_{b}^{-s}(u)=u
$$

for all positive integers $r$ and $s$.

Case 1. The geodesic $\tilde{u}$ is trivial on $\tilde{S}$. In this case, $u=\partial \Delta_{1}$ for a twice punctured disk $\Delta_{1}$ enclosing $z$. From Theorem 2 of [10] and Theorem 2 of [13], there are parabolic elements $h_{1}, h_{2} \in G$ such that

$$
h_{1}^{*}=t_{a} \quad \text { and } \quad h_{2}^{*}=t_{b} .
$$

Note that $\Delta_{1}$ is also a twice punctured disk enclosing $z$. There is a parabolic element $T_{1} \in G$ that corresponds to the Dehn twist $t_{\partial \Delta_{1}}$, i.e., $T_{1}^{*}=t_{\partial \Delta_{1}}=t_{u}$. But we know that $t_{b}=t_{f(a)}=$ $f \circ t_{a} \circ f^{-1}$. Hence $h_{2}=g h_{1} g^{-1}$. From (3.1) (by setting $r=s=1$ ) we obtain $t_{a} \circ f \circ$ $t_{a}^{-1} \circ f^{-1}(u)=u$, which tells us that the commutator $\left[h_{1}, g\right]=h_{1} g h_{1}^{-1} g^{-1}$ commutes with $T_{1}$. From Lemma 5.2 of [20], $\left[h_{1}, g\right]$ also fixes the fixed point of $T$, which says that $\left[h_{1}, g\right]$ and $T$ share a common fixed point. Clearly, $\left[h_{1}, g\right]$ is non-trivial (otherwise, $h_{1}$ commutes with $g$, a contradiction). Since $G$ is discrete, by Theorem 5.1 .2 of [1], $\left[h_{1}, g\right]$ cannot be hyperbolic. But on the other hand, by Theorem 7.39.1 of Beardon [1], for a parabolic element $h_{1}$, and a hyperbolic element $g$, the commutator $\left[h_{1}, g\right] \in G$ is always hyperbolic. This is a contradiction.

Case 2. The geodesic $\tilde{u}$ is non-trivial on $\tilde{S}$. Note that $\tilde{u}$ denotes the geodesic representative on $\tilde{S}$ homotopic to $u$ when $u$ is viewed as a curve on $\tilde{S}$. As discussed in Section 2 , we denote by ( $\left.\tau_{\hat{u}}, \Omega_{\hat{u}}, \mathscr{U}_{\hat{u}}\right)$ the configuration corresponding to $u$. The equality (3.1) yields that $\left(t_{a}^{r} \circ t_{b}^{-s}\right) \circ t_{u}=t_{u} \circ\left(t_{a}^{r} \circ t_{b}^{-s}\right)$. It follows from (3.1) and Lemma 2.3 that both $\left[h_{1}, g\right]$ and $h_{1}^{r} h_{2}^{-s}$ send each maximal element of $\mathscr{U}_{\hat{u}}$ to a maximal element. By the assumption, $g$ is an essential hyperbolic element of $G$ whose axis axis $(g)$ intersects one (and hence infinitely many) of the preimages $\left\{\varrho^{-1}(\tilde{u})\right\}$, say $\hat{u}_{0}$. Note that $\hat{u}_{0}$ could be the boundary of a maximal element of $\mathscr{U}_{\hat{u}}$, but $\hat{u}_{0}$ could also be a boundary of an element of $\mathscr{U}_{\hat{u}}$ of higher order. If the later occurs, axis $(g)$ is contained in a maximal element of $\mathscr{U}_{\hat{u}}$.

In each of the following cases we will show there is a maximal element $U_{0}$ of $\mathscr{U}_{\hat{u}}$ such that $h_{1}^{r} h_{2}^{-s}$ or its inverse $h_{2}^{s} h_{1}^{-r}$ does not send $U_{0}$ to a maximal element of $\mathscr{U}_{\hat{u}}$. But from (3.2), (3.1) and Lemma 2.3, $h_{1}^{r} h_{2}^{-s}$ and $h_{2}^{s} h_{1}^{-r}$ send every maximal element of $\mathscr{U}_{\hat{u}}$ to a maximal element of $\mathscr{U}_{\hat{u}}$, which will lead to a contradiction. 


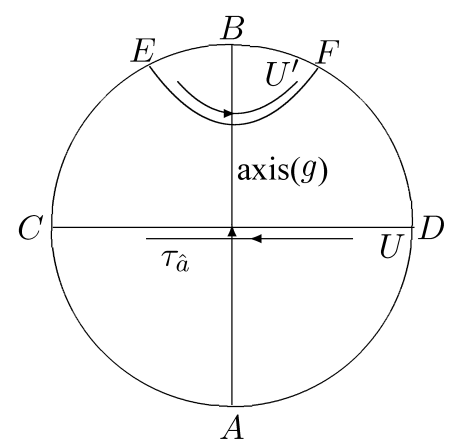

FIG. 2

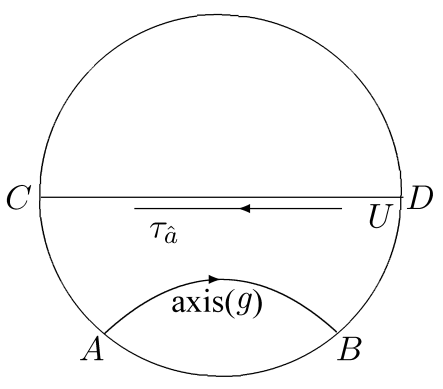

FIG. 3

Subcase 1. The geodesic $\hat{u}_{0}$ is the boundary of a maximal element $U \in \mathscr{U}_{\hat{u}}$. In this case, we may assume that $\hat{u}_{0}=\hat{u}=\partial U$ and that $U$ covers the repelling fixed point $A$ of $g$, as shown in Fig. 2.

In the rest of the article we use $(A C)$, for example, to denote the unoriented arc in $\mathbf{S}^{1}$ connecting the two labeling points $A$ and $C$ without passing through any other labeling points. Denote by $U^{\prime} \in \mathscr{U}_{\hat{u}}$ the other maximal element containing $g(\mathbf{D} \backslash U)$. Then $U^{\prime}$ covers the attracting fixed point $B$ of $g$. Let $x$ denote the fixed point of $h_{1}$. Then $g(x)$ is the fixed point of $h_{2}=g h_{1} g^{-1}$. We assume that $h_{1}$ points in the counterclockwise direction, and thus $h_{2}^{-1}$ points in the clockwise direction.

If $x \in(C E)$, then $g(x) \in(B E)$. As $\tilde{u}$ is simple, for sufficiently large integers $r$ and $s$, $h_{2}^{-s}\left(\mathbf{D} \backslash U^{\prime}\right) \cap \mathbf{S}^{1} \subset(E B)$, and thus $h_{1}^{r} h_{2}^{-s}\left(\mathbf{D} \backslash U^{\prime}\right) \subset \mathbf{D} \backslash U^{\prime}$. It follows from Lemma 4.3 of [19] that $h_{1}^{r} h_{2}^{-s}\left(U^{\prime}\right)$ is not a maximal element of $\mathscr{U}_{\hat{u}}$. This contradicts Lemma 2.3. The same argument applies to the case of $x \in(F D)$. If $x \in(E B)$, then since $B$ is the attracting fixed point of $g, g(x) \in(E B)$ is closer to $B$ than $x$. For large $r$ and $s, h_{2}^{-s}\left(\mathbf{D} \backslash U^{\prime}\right) \cap \mathbf{S}^{1} \subset(x g(x))$ is disjoint from $\mathbf{D} \backslash U^{\prime}$. It follows that $h_{1}^{r} h_{2}^{-s}\left(\mathbf{D} \backslash U^{\prime}\right)$ is disjoint from $\mathbf{D} \backslash U^{\prime}$. Hence by Lemma 4.3 of [19], $h_{1}^{r} h_{2}^{-s}$ and hence its inverse $h_{2}^{s} h_{1}^{-r}$ does not send $U^{\prime}$ to any maximal element of $\mathscr{U}_{\hat{u}}$. This again contradicts Lemma 2.3. The same is true when $x \in(B F)$.

If $x \in(C A)$, then since $A$ is repelling fixed point of $g$, either $g(x) \in(C A)$, or $g(x) \in$ $(C B)$. If $g(x) \in(C A)$, then $h_{2}^{-s}(\mathbf{D} \backslash U) \cap \mathbf{S}^{1} \subset(g(x) x)$, and thus $h_{1}^{r} h_{2}^{-s}(\mathbf{D} \backslash U) \subset(g(x) x)$. It follows that $\mathbf{D} \backslash U$ is disjoint from $h_{1}^{r} h_{2}^{-s}(\mathbf{D} \backslash U)$. Hence by Lemma 4.3 of [19], $h_{2}^{s} h_{1}^{-r}(U)$ is not a maximal element of $\mathscr{U}_{\hat{u}}$. This contradicts Lemma 2.3. If $g(x) \in(C E)$, then $h_{1}^{-r}(\mathbf{D} \backslash U) \cap$ $\mathbf{S}^{1} \subset(C A)$ and thus $h_{2}^{s} h_{1}^{-r}(\mathbf{D} \backslash U) \cap \mathbf{S}^{1} \subset(C E)$. We see that $h_{2}^{s} h_{1}^{-r}(\mathbf{D} \backslash U) \subset \mathbf{D} \backslash U$. This implies $U \subset h_{2}^{s} h_{1}^{-r}(U)$. In particular, $U$ is not a maximal element of $\mathscr{U}_{\hat{u}}$. If $g(x) \in(E B)$, then $h_{1}^{r} h_{2}^{-s}(U) \subset U$. This is also impossible. The same argument applies to the case of $x \in(A D)$.

Subcase 2. The axis axis $(g)$ is contained in a maximal element $U \in \mathscr{U}_{\hat{u}}$. See Fig. 3. If 
$x \in(A C)$, then $g(x)$ lies in $(A C),(C D)$ or $(B D)$. If $g(x)$ is in $(A C), g(x)$ is closer to $C$ than $x$. One sees that $h_{2}^{s} h_{1}^{-r}(\mathbf{D} \backslash U)$ is disjoint from $\mathbf{D} \backslash U$ for large $r$ and $s$. So $h_{2}^{s} h_{1}^{-r}$ and hence $h_{1}^{r} h_{2}^{-s}(U)$ is not a maximal element. If $g(x)$ is in $(C D)$, one checks that $h_{1}^{r} h_{2}^{-s}(U) \subset U$ for large $r$ and $s$, and this would imply that $h_{1}^{r} h_{2}^{-s}(U)$ is not a maximal element. If $g(x)$ is in $(B D), h_{2}^{s} h_{1}^{-r}(\mathbf{D} \backslash U)$ is disjoint from $\mathbf{D} \backslash U$ for large $r$ and $s$, which says that $U$ is not a maximal element of $\mathscr{U}_{\hat{u}}$.

If $x \in(B D)$ (resp. $x \in(A B)$ ), then since $B$ is the attracting fixed point of $g, g(x) \in$ $(B D)$ (resp. $g(x) \in(A B)$ ) is closer to $B$ than $x$. As one can see, $h_{1}^{r} h_{2}^{-s}(\mathbf{D} \backslash U)$ is disjoint from $\mathbf{D} \backslash U$. It follows that $h_{1}^{r} h_{2}^{-s}(U)$ is not a maximal element of $\mathscr{U}_{\hat{u}}$. Finally, if $x \in(C D)$, then $g(x) \in(B D)$. For large $r$ and $s, h_{2}^{s} h_{1}^{-r}(U) \subset U$. This tells us that $h_{2}^{s} h_{1}^{-r}(U)$ is not a maximal element of $\mathscr{U}_{\hat{u}}$.

This case-by-case argument finishes the proof of Theorem 1.1.

Proof OF COROLlary 1.1: Let $z, z_{1}, \ldots, z_{k}$ denote all the punctures contained in $D_{\alpha}$. Let $\Lambda_{\alpha}$ be the corresponding path connecting $z, z_{1}, \ldots, z_{k}$ in this order. Let $\Lambda_{\alpha}^{\prime}$ be the sub-path of $\Lambda_{\alpha}$ connecting $z$ and $z_{1}$. Let $\Delta_{\alpha}$ be a fattening of $\Lambda_{\alpha}^{\prime}$. Then $\Delta_{\alpha}$ is a twice punctured disk enclosing $z$. From Theorem 1.1, $\left(\partial \Delta_{\alpha}, f^{k}\left(\partial \Delta_{\alpha}\right)\right)$ fills $S$ for all $k \geq 1$. It is clear that $\left(\partial \Delta_{\alpha}, f\left(\partial \Delta_{\alpha}\right)\right)$ fills $\tilde{S}$ if and only if $\left(\Lambda_{\alpha}^{\prime}, f\left(\Lambda_{\alpha}^{\prime}\right)\right)$ fills $S$. Since $\Lambda_{\alpha}^{\prime} \subset \Lambda_{\alpha}$ and $f\left(\partial \Delta_{\alpha}\right)=\partial f\left(\Delta_{\alpha}\right)$, we see that $\left(\Lambda_{\alpha}, f\left(\Lambda_{\alpha}\right)\right)$ fills $\tilde{S}$. From the construction, $D_{\alpha}$ is a fattening of $\Lambda_{\alpha}$. We conclude that $(\alpha, f(\alpha))$ fills $S$, as asserted.

\section{Proof of Theorem 1.2}

By the assumption, we know that $\Delta$ and $\Delta_{0}$ enclose the same punctures $z$ and $z_{0}$ and that ( $\partial \Delta, \partial \Delta_{0}$ ) fills $S$. As $\partial \Delta$ and $\partial \Delta_{0}$ are loops around the same puncture $z_{0}$ of $\tilde{S}$ as $z$ is filled in, it is clear that the primitive parabolic elements $T$ and $T_{0}$ of $G$ corresponding to $\partial \Delta$ and $\partial \Delta_{0}$ are conjugate to each other in $G$. It follows that there is an element $h \in G$ sending the fixed point $x$ of $T$ to the fixed point $x_{0}$ of $T_{0}$. As such, $F=h^{*}$ sends $\partial \Delta$ to $\partial \Delta_{0}$. Of course, $F \in \mathscr{F}_{0}(S)$. We need to prove that $F \circ t_{\partial \Delta}^{-k}$ are pseudo-Anosov for either all $k>0$ or all $k<0$.

If $h$ is essential hyperbolic, then $F$ is pseudo-Anosov. Hence $F \in \mathscr{F}(S)$ and by Lemma 3.1 of [22], we conclude that $F \circ t_{\partial \Delta}^{-k}$ are pseudo-Anosov for all $k \geq 0$ or $k \leq 0$.

If $h$ is parabolic, then by Theorem 2 of $[10,13], F=t_{c}$ or $t_{c}^{-1}$, where $c$ is a simple closed geodesic that is also trivial on $\tilde{S}$, i.e., $c=\partial \Delta^{\prime}$ for some twice punctured disk $\Delta^{\prime}$ enclosing $z$. Assume that $F=t_{c}$. By the definition, $\partial \Delta_{0}=t_{c}(\partial \Delta)$. We see that

$$
t_{c} \circ t_{\partial \Delta} \circ t_{c}^{-1}=t_{\partial \Delta_{0}} .
$$

Since $\left(\partial \Delta, \partial \Delta_{0}\right)$ fills $S$, from $(4.1), c$ intersects $\partial \Delta$. We claim that $(\partial \Delta, c)$ also fills $S$. In fact, the geodesic $t_{c}(\partial \Delta)=\partial \Delta_{0}$ is homotopic to a closed curve that stays in an arbitrary small neighborhood $\mathscr{N}$ of $\partial \Delta \cup c$. If $(\partial \Delta, c)$ does not fill $S$, then there is a non-trivial loop 
$e$ that is disjoint from $\partial \Delta \cup c$. So $e$ is also disjoint from $\mathscr{N}$ if $\mathscr{N}$ is made to be sufficiently small. It follows that $e$ is disjoint from both $\partial \Delta$ and $\partial \Delta_{0}$, contradicting that $\left(\partial \Delta, \partial \Delta_{0}\right)$ fills $S$.

Hence by Thurston's theorem [14], $t_{c} \circ t_{\partial \Delta}^{-k}$ for all $k>0$ are pseudo-Anosov maps. Note also that both $c$ and $\partial \Delta$ are trivial on $\tilde{S}$ (that is, they are freely homotopic to a puncture of $\tilde{S}$ ) and $t_{c} \circ t_{\partial \Delta}^{-k}(\partial \Delta)=t_{c}(\partial \Delta)=\partial \Delta_{0}$, we see that $t_{c} \circ t_{\partial \Delta}^{-k} \in \mathscr{F}(S)$ sends $\partial \Delta$ to $\partial \Delta_{0}$.

It remains to consider the case where $h$ is non-essential hyperbolic and non-parabolic element of $G$. Recall that $h$ possesses the property that $\left(\partial \Delta, h^{*}(\partial \Delta)\right)$ fills $S$. Our aim is to show that $h^{*} \circ t_{\partial \Delta}^{-k}$ is pseudo-Anosov for either all $k>0$ or all $k<0$.

Suppose that for some $k>0$ and some $k<0$, there is a system $\Gamma$ (which depends on $k$ and is defined as in (2.1)) such that

$$
h^{*} \circ t_{\partial \Delta}^{-k}\left(\left\{u_{1}, \ldots, u_{s}\right\}\right)=\left\{u_{1}, \ldots, u_{s}\right\} .
$$

This tells us that

$$
\left(h^{*} \circ t_{\partial \Delta}^{-k}\right) \circ\left(t_{u_{1}} \circ \cdots \circ t_{u_{s}}\right)=\left(t_{u_{1}} \circ \cdots \circ t_{u_{s}}\right) \circ\left(h^{*} \circ t_{\partial \Delta}^{-k}\right) .
$$

There are two cases to consider.

Case 1. All $\tilde{u}_{i}, u_{i} \in \Gamma$, are non-trivial. Our first claim is that there is at least one $u=u_{i} \in \Gamma$, say, such that $u$ intersects $\partial \Delta$. Suppose to the contrary. That is, all $u_{i}$ are disjoint from $\partial \Delta$. Hence $t_{u_{1}} \circ \cdots \circ t_{u_{s}}$ commutes with $t_{\partial \Delta}$. From (4.2) we see that $h^{*}$ commutes with $t_{u_{1}} \circ \cdots \circ t_{u_{s}}$ and thus that

$$
\left(h^{*} \circ t_{\partial \Delta}^{-k} \circ\left(h^{*}\right)^{-1}\right) \circ\left(t_{u_{1}} \circ \cdots \circ t_{u_{s}}\right)=\left(t_{u_{1}} \circ \cdots \circ t_{u_{s}}\right) \circ\left(h^{*} \circ t_{\partial \Delta}^{-k} \circ\left(h^{*}\right)^{-1}\right) .
$$

On the other hand, since $\left(\partial \Delta, \partial \Delta_{0}\right)$ fills $S$, every $u_{i}$ must intersect $\partial \Delta_{0}$. This implies

$$
t_{\partial \Delta_{0}}^{-k} \circ\left(t_{u_{1}} \circ \cdots \circ t_{u_{s}}\right) \neq\left(t_{u_{1}} \circ \cdots \circ t_{u_{s}}\right) \circ t_{\partial \Delta_{0}}^{-k} .
$$

But $t_{\partial \Delta_{0}}^{-k}=h^{*} \circ t_{\partial \Delta}^{-k} \circ\left(h^{*}\right)^{-1}$. We see that

$$
\left(h^{*} \circ t_{\partial \Delta}^{-k} \circ\left(h^{*}\right)^{-1}\right) \circ\left(t_{u_{1}} \circ \cdots \circ t_{u_{s}}\right) \neq\left(t_{u_{1}} \circ \cdots \circ t_{u_{s}}\right) \circ\left(h^{*} \circ t_{\partial \Delta}^{-k} \circ\left(h^{*}\right)^{-1}\right) .
$$

This is absurd. We conclude that there is a geodesic $u \in \Gamma$ such that $u$ intersects $\partial \Delta$. Note that $h^{*} \circ t_{\partial \Delta}^{-k} \in \mathscr{F}_{0}(S)$.

Our next claim is that for any integer $m$,

$$
\left(h^{*} \circ t_{\partial \Delta}^{-k}\right)^{m}(u)=u \text {. }
$$

This assertion was implicitly proved in [10]. For completeness, however, the proof of (4.3) is included as follows. Since $h^{*} \circ t_{\partial \Delta}^{-k} \in \mathscr{F}_{0}(S)$, we let $h_{1} \in G$ be such that $h_{1}^{*}=h^{*} \circ t_{\partial \Delta}^{-k}$. From Theorem 2 of [13] and Theorem 2 of [10], we know that if $h_{1}$ is parabolic, then $h_{1}^{*}$ is 
represented by a power of a Dehn twist $t_{c}$ for a simple closed geodesic $c$ on $\dot{S}$. In this case, $h_{1}^{*}\left(u_{i}\right)=u_{i}$ for each $u_{i} \in \Gamma$. If $h_{1}$ is simple hyperbolic, then $h_{1}^{*}$ is represented by a power of a spin map $t_{\alpha}^{-1} \circ t_{\beta}$, where $\{\alpha, \beta\}$ forms the boundary of an $z$-punctured cylinder on $S$. In this case, we also see that $h_{1}^{*}\left(u_{i}\right)=u_{i}$ for each $u_{i} \in \Gamma$. If $h_{1}$ is non-simple and non-essential, then by Theorem 2 of [10], there is a unique pseudo-Anosov component $\mathscr{P} \subset S$ for $h_{1}^{*}$ that contains $z$. As it turns out, any curve $u_{i}$ in $\Gamma$ cannot meet $\mathscr{P}$ in a non-trivial way, which means that all $u_{i} \in \Gamma$ stays outside of $\mathscr{P}$. It follows that $h_{1}^{*}\left(u_{i}\right)=u_{i}$. Finally, if $h_{1}$ is essential hyperbolic, then by Theorem 2 of [10] again, $h_{1}^{*}$ is pseudo-Anosov. By the assumption, this case does not occur. We thus conclude that (4.3) holds for every $u \in \Gamma$.

Now let $\left(\tau_{\hat{u}}, \Omega_{\hat{u}}, \mathscr{U}_{\hat{u}}\right)$ be the configuration corresponding to $u$. From Lemma 2.2, there exists a maximal element $U \in \mathscr{U}_{\hat{u}}$ that covers $x$. Recall that $x$ is the fixed point of the parabolic element $T \in G$ that corresponds to $\partial \Delta$. If the axis axis $(h)$ is disjoint from $U$ (Fig. 4), then for any $k \neq 0, h T^{-k}(\mathbf{D} \backslash U) \subset \mathbf{D} \backslash U$. Hence $\left(h T^{-k}\right)^{m}(\mathbf{D} \backslash U) \subset \mathbf{D} \backslash U$. That is, $\left(h T^{-k}\right)^{m}(U)$ is not a maximal element of $\mathscr{U}_{\hat{u}}$. This contradicts Lemma 2.3.

Consider the case where axis $(h)$ crosses $U$. Let $U^{\prime} \in \mathscr{U}_{\hat{u}}$ be the other maximal element intersecting axis $(h)$ (Lemma 2.1 of [21]). See Fig. 5. If the attracting fixed point of $h$ is in $U$, that is, $A$ is the attracting fixed point of $h$, then $T^{-k}(\mathbf{D} \backslash U) \subset U$ and thus $h T^{-k}(\mathbf{D} \backslash U) \subset U$, which says $h T^{-k}(U) \cup U=\mathbf{D}$, contradicting Lemma 2.4.

Now we assume that the attracting fixed point of $h$ is in $U^{\prime}$. In this case, $U$ covers the repelling fixed point of $h$, denoted by $A$. Recall that the motion $T$ points in the counterclockwise direction (as shown in Fig. 5). Now the relative position between $x$ and $A$ determines whether we choose $k>0$ or $k<0$. We assume without loss of generality that $x$ is on the left side of $A$, as shown also in Fig. 5. By examining the action of $T^{-k}$ for any $k<0$, we find that the motion of $T^{-k}$ (for any $k<0$ ) and $h$ have the same relative motion direction. It turns out that

$$
T^{-k}(\mathbf{D} \backslash U) \cap \mathbf{S}^{1} \subset(C x) .
$$

Since $A$ is the repelling fixed point of $h, h T^{-k}(\mathbf{D} \backslash U)$ lies in either (i) $U$, or (ii) $U^{\prime}$, or

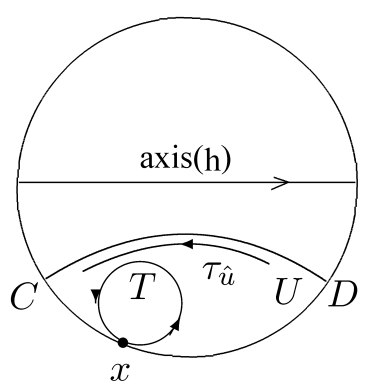

FIG. 4

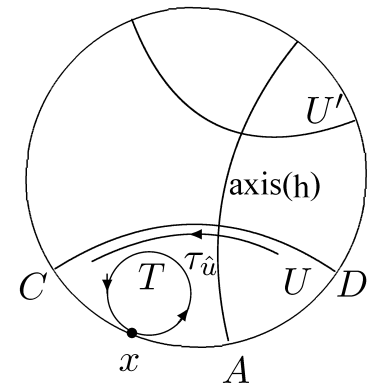

FIG. 5 
(iii) $\mathbf{D} \backslash\left(U \cup U^{\prime}\right)$. Notice that (i) implies that $h T^{-k}(U) \cup U=\mathbf{D}$, which would contradict Lemma 2.4. (ii) implies that $\left(h T^{-k}\right)^{i}\left(U^{\prime}\right) \subset U^{\prime}$ for all $i>0$, which says that $\left(h T^{-k}\right)^{i}\left(U^{\prime}\right)$ are never maximal elements of $\mathscr{U}_{\hat{u}}$. If (iii) holds, then one easily checks that for all $i>0,\left(h T^{-k}\right)^{i}(\mathbf{D} \backslash U) \subset \mathbf{D} \backslash\left(U \cup U^{\prime}\right)$. That is, $U \cup U^{\prime} \subset\left(h T^{-k}\right)^{i}(U)$. In other words, $\left(h T^{-k}\right)^{i}(U)$ never becomes a maximal element of $\mathscr{U}_{\hat{u}}$. From Lemma 2.3, we see that (4.3) never occurs.

REMARK. In the case where $k>0$, we observe that $T^{-k}(\mathbf{D} \backslash U)$ could possibly cover the repelling fixed point $A$ of $h$. If this occurs, then $h T^{-k}(\mathbf{D} \backslash U) \subset \mathbf{D} \backslash U^{\prime}$ and there is no guarantee that $h T^{-k}(U) \neq U, U^{\prime}$. Thus no contradiction can be found. Nevertheless, the above argument tells us that for all sufficiently large positive integers $k, h T^{-k}(U)$ are not maximal elements of $\mathscr{U}_{\hat{u}}$, which will lead to that $h T^{-k} \in \mathscr{F}(S)$ for sufficiently large $k$.

Case 2. There is one $u \in \Gamma$ such that $\tilde{u}$ is trivial. In this case, $u=\partial \Delta^{\prime}$ for some twice punctured disk enclosing $z$ and $u$ is the only one element in $\Gamma$ with $\tilde{u}$ being trivial. We have

$$
h^{*} \circ t_{\partial \Delta}^{-k}(u)=u \text {. }
$$

Let $y \in \mathbf{S}^{1}$ be the fixed point of the parabolic element corresponding to $u$. (4.4) then yields

$$
h T^{-k}(y)=y \text {. }
$$

This means that $h T^{-k}$ is also a parabolic element. Write $T_{u}=h T^{-k}$. From (4.5), we have $T_{u}^{*}=t_{u}$ or $t_{u}^{-1}$. Assume that $T_{u}^{*}=t_{u}$ (the case where $T_{u}^{*}=t_{u}^{-1}$ can be handled similarly and is omitted). Then

$$
h=T_{u} T^{k}, \quad \text { or } \quad h^{*}=t_{u} \circ t_{\partial \Delta}^{k} .
$$

Now consider the pair $(\partial \Delta, u)$. It is clear that $(\partial \Delta, u)$ does not fill $S$. Otherwise, by Thurston [14], $t_{u} \circ t_{\partial \Delta}^{k}$ for each $k<0$ would be a pseudo-Anosov map. It follows from (4.6) that $h^{*}$ is pseudo-Anosov. Hence by Theorem 2 of [10], $h$ is an essential hyperbolic element, contradicting the hypothesis.

We also know that $u$ must intersect $\partial \Delta$. Since $(\partial \Delta, u)$ does not fill $S$, there is a simple closed geodesic $v$ disjoint from $\partial \Delta \cup u$. The geodesic $\partial \Delta_{0}=h^{*}(\partial \Delta)$ is homotopic to the image curve $t_{u} \circ t_{\partial \Delta}^{k}(\partial \Delta)=t_{u}(\partial \Delta)$ that is defined in a neighborhood $\mathscr{N}$ of $\partial \Delta \cup u$, where $\mathscr{N}$ is chosen to be so small that $v$ is disjoint from $\mathscr{N}$. We conclude that $v$ does not intersect $\partial \Delta \cup \partial \Delta_{0}$. That is, $\left(\partial \Delta, \partial \Delta_{0}\right)$ does not fill $S$. This contradicts the hypothesis.

We conclude that $h^{*} \circ t_{\partial \Delta}^{-k}$, which sends $\partial \Delta$ to $\partial \Delta_{0}$, is pseudo-Anosov for either all $k>0$ or all $k<0$. This completes the proof of Theorem 1.2.

Proof of Corollary 1.2: Let $\left\{z, z_{0}\right\}$ and $\left\{z, z^{\prime}\right\}$ denote the punctures in $\Delta$ and $\Delta^{\prime}$, respectively. Suppose that such an $f$ exists and that $z_{0} \neq z^{\prime}$. As $f$ projects to a map $\tilde{f}$ on $\tilde{S}$, it is obvious that $f$ fixes the puncture $z$ and so $\tilde{f}\left(z_{0}\right)=z^{\prime}$, contradicting the fact that $\tilde{f}$ is 
isotopic to the identity on $\tilde{S}$. From Theorem 1.1, $f(\partial \Delta)=\partial \Delta^{\prime}$ and $\left(\partial \Delta, \partial \Delta^{\prime}\right)$ fills $S$. Hence $\Delta^{\prime} \in \mathscr{T}(\Delta)$.

Conversely, if $\Delta^{\prime} \in \mathscr{T}(\Delta)$, then by Theorem 1.2, there is an element $f \in \mathscr{F}(S)$ sending $\Delta$ to $\Delta^{\prime}$, as claimed.

\section{A classification of elements of $\mathscr{F}(S)$ in terms of $\mathscr{T}(\Delta)$}

To prove Theorem 1.3, we need the following lemma.

Lemma 5.1. Let $F: S \rightarrow S$ be obtained from Theorem 1.2. Then every element $\mathscr{F}(S)$ that sends $\partial \Delta$ to $\partial \Delta_{0}$ is of the form $F \circ t_{\partial \Delta}^{-k}$ for some integer $k$.

Proof. Let $f \in \mathscr{F}(S)$ be such that $f(\partial \Delta)=\partial \Delta_{0}$. Note that $\mathscr{F}_{0}(S)$ is the kernel of the group homomorphism of $\operatorname{Mod}_{S}^{z}$ onto $\operatorname{Mod}(\tilde{S})$. There is an essential hyperbolic element $g \in G$ so that $g^{*}=f$. Also, as mentioned earlier, the parabolic elements $T$ and $T_{0}$ of $G$ that correspond to $\partial \Delta$ and $\partial \Delta_{0}$ are conjugate to each other. Hence there is an element $F \in \mathscr{F}_{0}(S)$ sending $\partial \Delta$ to $\partial \Delta_{0}$. Recall that $F=h^{*}$ for some element $h \in G$.

Observe that $h(x)=x_{0}$ is the fixed point of $T_{0}=h T h^{-1}$. On the other hand, since $f(\partial \Delta)=\partial \Delta_{0}, f \circ t_{\partial \Delta} \circ f^{-1}=t_{\partial \Delta_{0}}$. It follows that $g T g^{-1}=T_{0}$, which implies $g(x)$ is the fixed point of $T_{0}$. But $T_{0}$ is parabolic, it has unique fixed point $x_{0}$ on $\mathbf{S}^{1}$. We conclude that $g(x)=h(x)$ or $g^{-1} h(x)=x$. If $g=h$, then $h$ is essential hyperbolic and thus $F$ is pseudoAnosov. Otherwise, $g^{-1} h$ is non-trivial. Since $T$ is parabolic, it also has a unique fixed point $x$ on $\mathbf{S}^{1}$. Hence $g^{-1} h$ and $T$ share the same fixed point $x$. In particular, $g^{-1} h$ cannot be hyperbolic (otherwise, $G$ would not be discrete) and the only possibility is that $g^{-1} h$ is also parabolic (if it is non-trivial) and so there is an integer $k$ such that $g^{-1} h=T^{k}$ or $g=h T^{-k}$. That is, $f=h^{*} \circ t_{\partial \Delta}^{-k}$.

Proof of Theorem 1.3: $\quad$ Let $f \in \mathscr{F}(S)$. By Theorem 1.1, $(\partial \Delta, \partial f(\Delta))$ fills $S$. Note that $f$ is isotopic to the identity on $\tilde{S}, \Delta$ and $f(\Delta)$ both enclose $z$ and $z_{0}$. Thus $f(\Delta) \in \mathscr{T}(\Delta)$. Since $f \circ t_{\partial \Delta}^{k}(\partial \Delta)=f(\partial \Delta)$ for any $k$, we obtain a map $\omega: \mathscr{F}(S) / \sim \rightarrow \mathscr{T}(\Delta)$.

Conversely, let $\Delta_{0} \in \mathscr{T}(\Delta)$. Then by the definition of $\mathscr{T}(\Delta),\left(\partial \Delta_{0}, \partial \Delta\right)$ fills $S$. By Theorem 1.2, there is $F \in \mathscr{F}_{0}(S)$ such that $F(\partial \Delta)=\partial \Delta_{0}$. Let $\chi\left(\Delta_{0}\right)$ be the $\Delta$-equivalence class of $F \circ t_{\partial \Delta}^{k}$. By Theorem 1.2, $F \circ t_{\partial \Delta}^{k}$ are pseudo-Anosov for either all $k>0$ or $k<0$. We thus obtain the map $\chi: \mathscr{T}(\Delta) \rightarrow \mathscr{F}(S) / \sim$.

We claim that $\chi \circ \omega=\mathrm{id}$ (which says that $\omega$ is injective). Indeed, for any $f \in \mathscr{F}(S)$, let $[f]_{\Delta}$ denote the $\Delta$-equivalence class of $f$ in $\mathscr{F}(S) / \sim$. By Theorem 1.1, $(\partial \Delta, f(\partial \Delta))$ fills $S$. By Theorem 1.2, there is $F$ sending $\Delta$ to $f(\Delta)$. From Lemma 5.1, $f=F \circ t_{\partial \Delta}^{k}$ for some $k$, which says that $\chi \circ \omega(f)$ is $\Delta$-equivalent to $f$. It follows that $\chi \circ \omega=$ id.

Finally, we prove that $\omega \circ \chi=\mathrm{id}$ (which says that $\omega$ is surjective). Let $\Delta_{0} \in \mathscr{T}(\Delta)$. Then $\left(\partial \Delta, \partial \Delta_{0}\right)$ fills $S$. By Theorem 1.2 again, there is $F \in \mathscr{F}_{0}(S)$ such that $F(\Delta)=\Delta_{0}$ and that $f:=F \circ t_{\partial \Delta}^{k}$ are pseudo-Anosov for all $k>0$ or $k<0$. This implies that $[f]_{\Delta}=\chi\left(\Delta_{0}\right)$. 
But since $f \circ t_{\partial \Delta}^{k}(\partial \Delta)=\partial \Delta_{0}$ for any $k$, we have $\omega \circ \chi\left(\Delta_{0}\right)=\Delta_{0}$, and thus $\omega \circ \chi=\mathrm{id}$, as claimed.

\section{Distances between elements of $\mathscr{T}(\Delta)$ and dilatations of associated pseudo- Anosov maps}

Proof of TheOREM 1.4: (1) From Theorem 1.3, we know that there is $f \in \mathscr{F}(S)$ such that $f(\Delta)=\Delta_{0}$. By Theorem 1.2, $f \circ t_{\partial \Delta}^{k}$ are pseudo-Anosov for either $k>0$ or $k<0$. We assume that $k>0$. It is clear that for all $k>0, f \circ t_{\partial \Delta}^{k}(\Delta)=\Delta_{0}$. We need to show that $\lambda\left(f \circ t_{\partial \Delta}^{k}\right) \rightarrow+\infty$ as $k \rightarrow+\infty$.

Note that $f \circ t_{\partial \Delta}^{k} \in \mathscr{F}(S)$ for any $k$. Let $\gamma, \gamma_{k}$ denote the filling closed geodesics on $\tilde{S}$ corresponding to $f$ and $f \circ t_{\partial \Delta}^{k}$, and let $i_{\gamma}$ and $i_{\gamma_{k}}$ denote the number of self-intersection points of $\gamma$ and $\gamma_{k}$, respectively. Assume that $z \in \gamma$. As $\Delta$ determines a path $\Lambda$ joining $z$ and $z_{0}, \Delta$ in turn determines a parabolic element $\delta \in \pi_{1}(\tilde{S}, z)$ around $z_{0}$.

By the same argument of Theorem 1.1 of [22], the curve concatenation $\delta^{k} \cdot \gamma$ is freely homotopic to $\gamma_{k}$, where we note that $\gamma_{k}$ is a filling closed geodesic. The associated homotopy is denoted by $\delta^{k} \cdot \gamma \sim \gamma_{k}$. Observe that the $k$-th power of $\delta$ repeats $\delta k$ times. During the deformation $\delta^{k} \cdot \gamma \sim \gamma_{k}$, a new set $I_{k}$ of self-intersection points of $\delta^{4}$ emerges. Fig. 6 below illustrates this process.

Fig. 6 (a) shows the multi-curve $\delta^{4}$ as a portion in the curve concatenation $\delta^{4} \cdot \gamma$. As we see, the multiplicity of $\delta$ is 4 . Fig. 6 (b) shows what $\delta^{4}$ looks like as a portion of $\gamma_{4}$, after the deformation $\delta^{4} \cdot \gamma \sim \gamma_{4}$ is performed. We see that the set of self-intersection points $I_{4}=\left\{p_{1}, p_{2}, p_{3}\right\}$ emerges.

We observe that any two points in $I_{k}$ cannot cancel each other, while since $i_{\gamma}$ is finite, only finite number of points in $I_{k}$ could possibly cancel some existing self-intersection points of $\gamma$. But note that the cardinality of $I_{k}$ tends to $+\infty$ as $k \rightarrow+\infty$, we conclude that $i_{\gamma_{k}} \rightarrow$

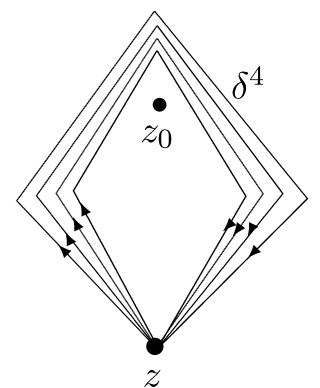

(a)

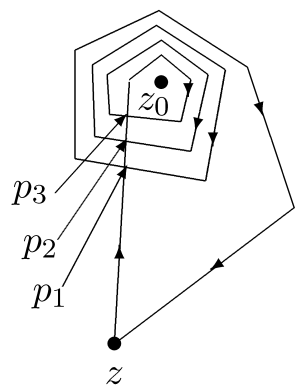

(b)

FIG. 6 
$+\infty$ as $k \rightarrow+\infty$. Since $f \circ t_{\partial \Delta}^{k} \in \mathscr{F}(S)$, from the argument of Theorem 1.1 of [22], we obtain $\lambda\left(f \circ t_{\partial \Delta}^{k}\right) \rightarrow+\infty$ as $k \rightarrow+\infty$. Since $f \sim f \circ t_{\partial \Delta}^{k}$ and $f \circ t_{\partial \Delta}^{k}(\Delta)=\Delta_{0}$ for any $k$, we are done. The proof of (2) is the same as that of Theorem 1.3 in [23].

\section{Examples}

In [16] we constructed an example demonstrating that for any twice punctured disk $\Delta$ that encloses $z$ and $z_{0}$, there are parabolic elements $h \in G$ such that $\left(\partial \Delta, h^{*}(\partial \Delta)\right)$ fills $S$. In the example below, we present a simple hyperbolic element $h \in G$ such that $\left(\partial \Delta, h^{*}(\partial \Delta)\right)$ fills $S$ and $h^{*}(\partial \Delta) \in \mathscr{T}(\Delta)$.

Note that $\Delta$ is a twice punctured disk on $S$ enclosing $z$; its deformation retract $\Lambda$ is a path connecting $z$ and another puncture $z_{1}$, say. The following constructions are suggested by the referee's comments on [18]. The surface $S$ can be thought of as a surface with $p$ handles $H_{1}, \ldots, H_{p}$ and $n+1$ punctures $z, z_{1}, \ldots, z_{n}$, where each handle is a copy of the handle $H$ drawn in Fig. 7.

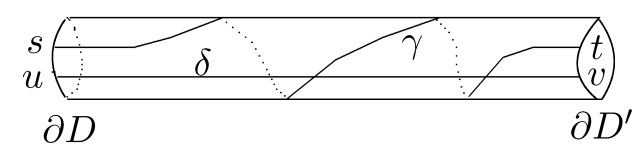

FIG. 7

$H$ has two boundary components $\left\{\partial D, \partial D^{\prime}\right\}$. Let $\gamma, \delta$ be two curves on $H$ that are not homotopic to each other and fill $H$. Let $\{s, t\}$ and $\{u, v\}$ are endpoints of $\gamma$ and $\delta$, respectively.

We remove from the sphere $\mathbf{S}^{2} p$ pairs of small disks $\left(D_{i}, D_{i}^{\prime}\right)$ and $z, z_{1}, \ldots, z_{n}$. Then the surface $S$ can be restored from attaching $p$ handles along the boundary components $\partial D_{i} \cong$ $\partial D$ and $\partial D_{i}^{\prime} \cong \partial D^{\prime}$ for $i=1,2, \ldots, p$.

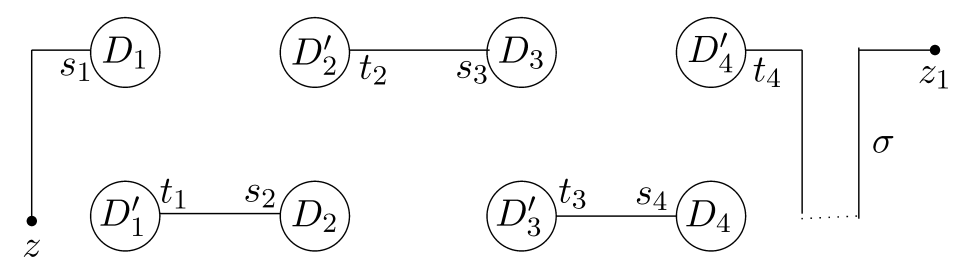

FIG. 8

Without loss of generality, we let $\Lambda$ (the deformation retract of $\Delta$ ) be the path described as follows. Connect $z$ and $s_{1}$, followed by $\gamma$ on $H_{1}$, then connect $t_{1}$ and $s_{2}$, and followed by 
$\gamma$ on $H_{2}$, and so forth. After $p$ steps, we connect $t_{p}$ and $z_{1}$ by a path $\sigma$ that is away from all other punctures. Fig. 8 shows a path $\Lambda$ in the case of $p=4$.

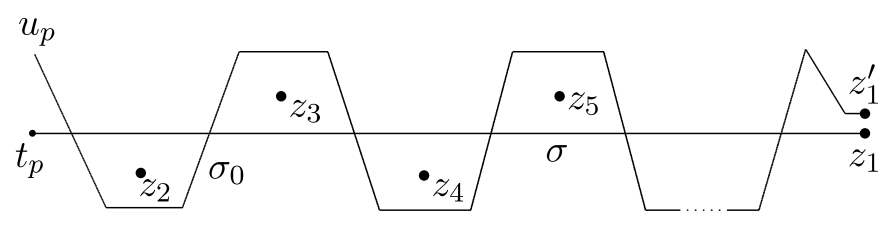

FIG. 9

Now we proceed to acquire a simple closed geodesic $C$ as follows. Choose a point $z^{\prime}$ that is near to $z$, connect $z^{\prime}$ and $v_{1}$, followed by the inverse $\delta^{-1}$ of $\delta$ on $H_{1}$, then connect $u_{1}$ and $v_{2}$, followed by $\delta^{-1}$ on $H_{2}$, then connect $u_{2}$ and $v_{3}$, and so forth (see Fig. 10). After $p$ steps, we draw a path $\sigma_{0}$ connecting $u_{p}$ to a point $z_{1}^{\prime}$ that is near to the puncture $z_{1}$ in such a way that $\mathbf{S}^{2} \backslash\left\{\sigma, \sigma_{0}\right\}$ are $n-1$ once punctured disks each of which contains only one puncture in $\left\{z_{2}, z_{3}, \ldots, z_{n}\right\}$. See Fig. 9.

Finally, we connect $z_{1}^{\prime}$ and $z^{\prime}$ (the point we begin with) by a path away from all holes $D_{i}, D_{i}^{\prime}$ and all punctures $z, z_{1}, \ldots, z_{n}$. Fig. 10 shows an example for such a simple closed curve $C$ in a surface of genus $p=4$. We thus obtain a simple closed curve $C$ on $S$ so that the graph $C \cup \Lambda$ fills $S$, i.e., $S \backslash C \cup \Lambda$ consists of polygons and possibly once punctured polygons.

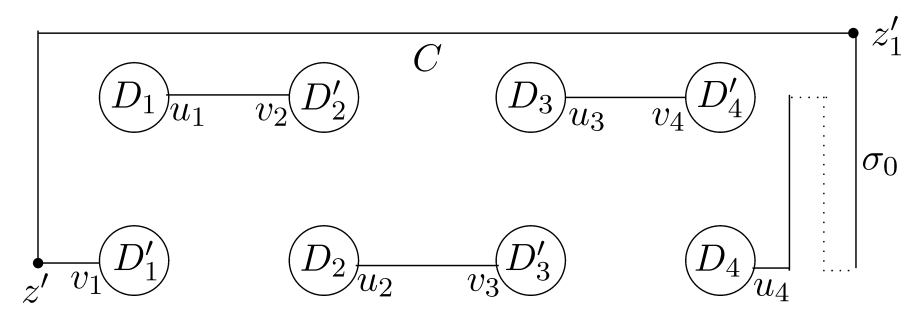

FIG. 10

Let $C_{0} \subset S$ be another simple closed curve so that $\left\{C, C_{0}\right\}$ are boundary components of a punctured cylinder $\mathscr{P}$ with puncture $z$. Clearly, $\left\{C, C_{0}\right\} \cup \Lambda$ fills $S$. There exists a simple hyperbolic element $h \in G$ so that $h^{*}=t_{C_{0}} \circ t_{C}^{-1}$. Note also that $\Delta$ can be restored from $\Lambda$ by a fattening process. We see that $\partial \mathscr{P} \cup \partial \Delta$ fills $S$. Let $\Delta_{0}=h^{*}(\Delta)$.

Proposition 7.1. The pair $\left(\partial \Delta, \partial \Delta_{0}\right)$ fills $S$. 
Proof. Assume that $\left(\partial \Delta, \partial \Delta_{0}\right)$ does not fill $S$. There is a geodesic $u \subset S$ such that $t_{\partial \Delta_{0}} \circ t_{\partial \Delta}^{-1}(u)=u$. Let $T \in G$ be the parabolic element corresponding to $t_{\partial \Delta}$. If $\tilde{u}$ is trivial, then the commutator $[h, T]=h T h^{-1} T^{-1}$ fixes a parabolic fixed point of $G$, so $[h, T]=h T h^{-1} T^{-1}$ is parabolic (otherwise $G$ would not be discrete). This contradicts that $[h, T]$ is hyperbolic.

If $\tilde{u}$ is non-trivial, by Lemma $2.3,[h, T]$ sends every maximal element $U \in \mathscr{U}_{\hat{u}}$ to a maximal element. On the other hand, we know from the hypothesis that $u$ is disjoint from $\partial \Delta$. By Lemma 2.2, the fixed point $x$ of $T$ must lie in $\Omega_{\hat{u}} \cap \mathbf{S}^{1}$. Since $\left\{C, C_{0}\right\} \cup \Lambda$ fills $S$ and $u$ is disjoint from $\partial \Delta, u$ must intersect $\left\{C, C_{0}\right\}$. By Lemma 2.1, axis $(h)$ crosses a maximal element $U \in \mathscr{U}_{\hat{u}}$. Let $U^{\prime} \in \mathscr{U}_{\hat{u}}$ be the other maximal element intersecting axis $(h)$ (by Lemma 2.1 of [21]).

We are thus in the situation of Fig. 2 (with $\operatorname{axis}(g)$ being replaced by axis $(h)$ ). Since $x \notin\left(U \cup U^{\prime}\right) \cap \mathbf{S}^{1}, x \in(C E) \cup(F D)$. Let us assume that $x \in(C E)$. By examining the action of the commutator $[h, T]$ on $U^{\prime}$, we find that $[h, T]\left(U^{\prime}\right) \subset U^{\prime}$, which says that $[h, T]\left(U^{\prime}\right)$ is not a maximal element of $\mathscr{U}_{\hat{u}}$. This is a contradiction.

ACKNOWLEDGMENT. The author is deeply grateful to the referees for carefully reading of the manuscript and the helpful suggestions. Their critical comments have enabled the author improve the paper in several aspects.

\section{References}

[ 1 ] BeARdon, A., The geometry of Discrete groups, Springer-Verlag, NY Heidelberg Berlin, 1983.

[ 2 ] BERS, L., Fiber spaces over Teichmüller spaces, Acta Math. 130 (1973), 89-126.

[ 3 ] Bers, L., An extremal problem for quasiconformal mappings and a theorem by Thurston, Acta Math. 141 (1978), 73-98.

[ 4 ] Birman, J. S., Braids, Links and Mapping class groups, Ann. of Math. Studies, No. 82, Princeton University Press, 1974.

[ 5 ] Boyer, S., Gordon, C. and Zhang, X., Dehn fillings of large hyperbolic 3-manifolds, J. Differential Geom. 58 (2001), No. 2, 263-308.

[ 6 ] FATHI, A., Dehn twists and pseudo-Anosov diffeomorphisms, Invent. Math. 87 (1987), 129-151.

[ 7 ] Fathi, A., Laudenbach, F. and Poenaru, V., Travaux de Thurston sur les surfaces, Seminaire Orsay, Asterisque, 66 and 67, Soc. Math. de France, (1979).

[ 8 ] Ivanov, N. V., Coefficients of expansion of pseudo-Anosov homeomorphisms, Zap. Nauchn. Sem. Leningrad. Otdel. Mat. Inst. Steklov. (LOMI), 167 (Issled. Topol. 6) 191 (1988), 111-116.

[ 9 ] Harvey, W. J., Boundary structure of the modular group, In Riemann surfaces and related topics: Proceedings of the 1978 Stony Brook Conference, Vol. 97 of Ann. of Math. Stud. 245-251, Princeton, N.J., (1981), Princeton Univ. Press.

[10] KrA, I., On the Nielsen-Thurston-Bers type of some self-maps of Riemann surfaces, Acta Math. 146 (1981), 231-270.

[11] Masur, H. and Minsky, Y., Geometry of the complex of curves I: Hyperbolicity, Invent. Math. 138 (1999), $103-149$.

[12] Long, D. D. and Morton, H., Hyperbolic 3-manifolds and surface homeomorphism, Topology 25 No. 4 (1986), 575-583. 
[13] NAG, S., Non-geodesic discs embedded in Teichmüller spaces, Amer. J. Math. 104 (1982), 339-408.

[14] Thurston, W. P., On the geometry and dynamics of diffeomorphisms of surfaces, Bull. Amer. Math. Soc. (N.S.) 19 (1988), 417-431.

[15] Zhang, C., Commuting mapping classes and their actions on the circle at infinity, Acta Math Sinica 52 (2009), 471-482.

[16] ZHANG, C., Some remarks on Thurston's constructions of pseudo-Anosov mapping classes of Riemann surfaces, Chinese Ann. of Math. Ser. B 29 (2008), 85-94.

[17] ZHANG, C., Pseudo-Anosov maps and fixed points of boundary homeomorphisms compatible with a Fuchsian group, Osaka J. Math. 46 (2009), 783-798.

[18] ZHANG C., Hyperbolic lengths of some filling geodesics on Riemann surfaces with punctures, Osaka J. Math. 45 (2008), 773-787.

[19] ZHANG, C., On products of pseudo-Anosov maps and Dehn twists of Riemann surfaces with punctures, J. Aust. Math. Soc. 88 (2010), 413-428.

[20] Zhang, C., Singularities of quadratic differentials and extremal Teichmüller mappings defined by Dehn twists, J. Aust. Math. Soc. 3 (2009), 275-288.

[21] ZHANG, C., Pseudo-Anosov maps and pairs of filling simple closed geodesics on Riemann surfaces, Tokyo J. Math. 35 (2012), 469-482.

[22] ZHANG, C., On the minimum dilatations of point-pushing pseudo-Anosov maps on Riemann surfaces with punctures, Preprint (2012).

[23] Zhang, C., On distances between curves in the curve complex and point-pushing pseudo-Anosov homeomorphisms, JP Journal of Geometry and Topology 12 (2012), 173-206.

Present Address:

DePARTMENT OF MATHEMATiCs,

Morehouse College,

Atlanta, GA 30314, USA. 